\title{
PROMINENCES IN ACTIVE REGIONS
}

\author{
J. KLECZEK \\ (Astronomical Institute Ondřejov, C.S.S.R.)
}

As an interplay of plasma and magnetic field the solar prominences represent a nice example of cosmical hydromagnetics. Due to stronger magnetic fields in active regions the prominence processes are more violent there than in non-active regions. Observing with a coronagraph or watching prominence movies one recognizes some characteristic processes in prominences of active regions:

(a) Condensation of $\mathrm{H} \alpha$ material in coronal space without any direct observational evidence of the material supply. It seems that the material had been ejected in the form of corpuscular radiation, captured, thermalized and cooled down to $10^{4}{ }^{\circ} \mathrm{K}$, when it becomes observable in the $\mathrm{H} \alpha$ line.

(b) Dissolution of the $\mathrm{H} \alpha$ material. Knots, streamers of sprays, surges and puffs often undergo a reverse process: they dissolve and disappear when observed in $\mathrm{H} \alpha$. Similar dissolution is typical for a great part of the material in eruptive prominences.

(c) Spiraling motion and detwisting of streamers are observed in surges, sprays, funnels and also in eruptive prominences. In the prominence movies of HAO Boulder, Sacramento Peak Observatory and Pic-du-Midi we have found many nice examples. In most cases (if not in all) the detwisting resulted in a marked simplification of the prominence structure.

(d) Emerging magnetic fields may be seen in form of small rising and expanding $\mathrm{H} \alpha$ loops (velocities about $50 \mathrm{~km} / \mathrm{sec}$ ). In this type of loops the material is dragged out of the chromosphere. A similar process with low velocities has been observed in $5303 \AA$ movies of coronal condensations.

(e) Capture of a surge by transversal magnetic fields has been observed and photographed on October 7,1956 . Surge material has been captured in a complex loopy structure and then streamed sunwards along the loops. This event shows that capturing of plasma streamers by transversal magnetic fields does occur on the Sun and that the material supply for $A S$ prominences (see item (a)) would thus be possible.

(f) Collisional processes occur in different forms. One has been just described in item (e). However, the well-known prominence of Lyot from 1937 shows that a surge hits a prominence without being (completely) captured. The prominence which has been hit decays and the surge material seems to be reflected (at least partly) downwards. Two colliding knots may bypass each other in complicated trajectories. Cases have been recorded of falling prominence knots being stopped and accelerated upwards, with measured acceleration more than 10 solar $\mathrm{g}$.

Kiepenheuer (ed.), Structure and Development of Solar Active Regions, 280-281. C. I.A.U. 
(g) Oscillating prominences at the limb have been photographed. The oscillations followed shortly after a nearby flare-spray event. This may be the counterpart of the disk phenomenon discovered by astronomers of Lockheed Observatory.

To explain the above-mentioned prominence processes, hydromagnetic laws are necessary. But before going into their theoretical explanation, more observational facts are needed. It has been the aim of my communication and of the movies demonstrating the processes described here, to call the attention of solar observers to the eventful prominence activity in solar active regions.

\section{DISCUSSION}

Sturrock: I am intrigued by the statement that the plasma of a surge is captured in closed magneticfield lines. It is difficult for a plasma to behave this way. Is there evidence that the field lines are closed before the surge, or only after the surge?

Kleczek: Yes, there is a clear observational evidence for that. The capturing of plasma in a transverse magnetic field has been proved experimentally (e.g. in laboratories of the U.S.S.R. and France) and the penetration of solar plasma into the Earth's magnetosphere is an example in cosmical scale.

H.U. Schmidt: I wonder whether it would not be possible to interpret these phenomena as a wave motion spread over a much larger solid angle than is covered by the surge material visible in $\mathrm{H} \alpha$ ? I would expect that the downstreaming matter in the loops is rather compressed in situ by a wave than trapped from the surge.

Kleczek: But in this case we must explain where the surge material $\left(\sim 10^{14}-10^{15}\right.$ grams $)$ disappears. As far as I may estimate, the amount of the material ejected in the surge is equal to the bright ('captured') material streaming sunwards in the loops.

Ohman: I would like to give reference in this connection to some observations made by Dr. L. Liszka at our observatory on Capri. Liszka found some similar disturbances by surges, even when the surges did not reach the prominence.

Kleczek: Yes, also in the prominence film that I have shown, the spray had not touched the neighbouring quiescent prominences and they began to oscillate. 